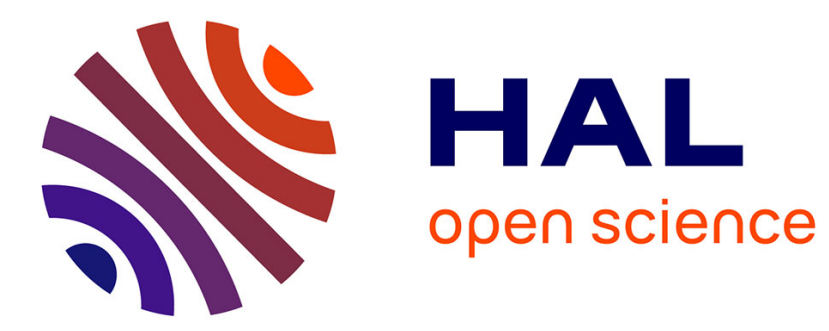

\title{
Solving the hypersingular boundary integral equation for the Burton and Miller formulation
}

\author{
Christophe Langrenne, Alexandre Garcia, Marc Bonnet
}

\section{To cite this version:}

Christophe Langrenne, Alexandre Garcia, Marc Bonnet. Solving the hypersingular boundary integral equation for the Burton and Miller formulation. Journal of the Acoustical Society of America, 2015, 138 (3332-3340), 10.1121/1.4935134 . hal-01233994

\section{HAL Id: hal-01233994 \\ https://hal.science/hal-01233994}

Submitted on 26 Nov 2015

HAL is a multi-disciplinary open access archive for the deposit and dissemination of scientific research documents, whether they are published or not. The documents may come from teaching and research institutions in France or abroad, or from public or private research centers.
L'archive ouverte pluridisciplinaire HAL, est destinée au dépôt et à la diffusion de documents scientifiques de niveau recherche, publiés ou non, émanant des établissements d'enseignement et de recherche français ou étrangers, des laboratoires publics ou privés. 


\title{
Solving the hypersingular boundary integral equation for the Burton and Miller formulation
}

\author{
Christophe Langrenne ${ }^{\mathrm{a})}$ and Alexandre Garcia \\ Laboratoire de Mécanique des Structures et des Systèmes Couplés (LMSSC) \\ Conservatoire National des Arts et Métiers \\ 292 rue Saint Martin, 75141 Paris Cedex 03 \\ Marc Bonnet \\ POEMS (ENSTA ParisTech, CNRS, INRIA, Université Paris-Saclay) \\ 828 Boulevard des Maréchaux, 91762 Palaiseau Cedex
}

\begin{abstract}
This paper presents an easy numerical implementation of the Burton and Miller (BM) formulation, where the hypersingular Helmholtz integral is regularized by identities from the associated Laplace equation, and thus needing only the evaluation of weakly singular integrals. The Helmholtz equation and its normal derivative are combined directly, with combinations at edge or corner collocation nodes not used when the surface is not smooth. The hypersingular operators arising in this process are regularized and then evaluated by an indirect procedure based on discretized versions of the Calderón identities linking the integral operators for associated Laplace problems. The method is valid for acoustic radiation and scattering problems involving arbitrarily shaped three-dimensional bodies. Unlike other approaches using direct evaluation of hypersingular integrals, collocation points still coincide with mesh nodes, as is usual when using conforming elements. Using higher-order shape functions (with the BEM model size kept fixed) reduces the overall numerical integration effort while increasing the solution accuracy. To reduce the condition number of the resulting $\mathrm{BM}$ formulation at low frequencies, a regularized version $\alpha=\mathrm{i} k /\left(k^{2}+\lambda\right)$ of the classical BM coupling factor $\alpha=\mathrm{i} / k$ is proposed. Comparisons with the CHIEF method of Schenck are made for four example configurations, two of them featuring non-smooth surfaces.
\end{abstract}

\footnotetext{
a) e-mail: christophe.langrenne@cnam.fr
} 


\section{Introduction}

The boundary element method (BEM) based on the Helmholtz integral equation (HIE) has long been applied for the analysis of acoustic radiation or scattering problems (direct problems) and source characterization (inverse problems). Its mathematical properties are extensively discussed $^{1 ; 2}$. When considering exterior Neumann problems, the solution of this formulation is non-unique for frequencies corresponding to eigenvalues of the associated interior Dirichlet problems ${ }^{3}$. This non-uniqueness is purely a drawback of the mathematical formulation of the problem and has no physical significance. To overcome this non-uniqueness problem within a direct BEM approach, two major formulations can be used: the Combined Helmholtz Integral Equation Formulation (CHIEF) proposed by Schenck $^{3}$ and the Coupled Helmholtz Integral (CHI) presented by Burton and Miller ${ }^{4}$.

CHIEF relies on an overdetermined system of equations generated using both surface and interior collocation points. As the latter points must not lie on a nodal surface of an interior standing wave, the selection of their number and location remains a problem. Wu improved the CHIEF method using a small interior region rather than a single point ${ }^{5}$.

The CHI method uses a linear combination of the HIE and its normal derivative. It is proved $^{8}$ that such a linear combination provides unique solutions at all frequencies, provided the imaginary part of the coupling factor is non-zero. Moreover, numerical experiments ${ }^{6}$ show that the eigenvalues of the relevant integral operator are moved away from the real axis by the CHI, and optimal selection of the coupling parameter is studied in ${ }^{7}$. The normal derivative of the HIE features a hypersingular integral operator, which makes this approach more difficult to implement than the usual HIE. Burton and Miller ${ }^{4}$ considered two methods for handling the hypersingular kernel. The first uses a double surface integral to reduce the order of the hypersingularity, which increases the numerical quadrature work. The second consists in expressing the hypersingular kernel using tangential derivatives on the surface. This approach is applied using planar elements ${ }^{9 ; 10}$ or quadratic quadrilateral isoparametric elements ${ }^{11}$. Another tangential formulation is used by $\mathrm{Wu}$ in an isoparametric element environment ${ }^{12}$. Cunefare modified the Burton and Miller formulation by taking interior additional collocation points, avoiding any singularity ${ }^{13}$.

An alternative way of dealing with the hypersingular operator arising in the CHI exploits known auxiliary solutions to the Laplace equation, leading to identities involving the static Green's function that allow to regularize the hypersingular integrals. Collocation BEM codes for Neumann acoustic problems are traditionally based on $C^{0}$ boundary elements (where nodal unknowns are defined at geometrical nodes), a setting adopted in this work as it fits our current implementation. However, later investigations on discontinuous, i.e. non-conforming, interpolations ${ }^{14}$ show considerable merit for this alternative treatment. The use of $C^{0}$ boundary elements in hypersingular operators presents a problem because the unit normal is usually not well-defined at nodes, due to the jump in the derivatives of the interpolating functions at element boundaries. Analytical formulations require collocation points interior to the elements for the unit normal to be well-defined there (whereas the HIE is normally collocated at element nodes). The two systems cannot, therefore, be combined directly as in the original Burton and Miller formulation. To perform this combination, interior pressure and velocity data must be interpolated from corresponding quantities at the element nodes. The choice of these interior points is crucial for the accuracy of the solution ${ }^{15}$. When an interior point is close to an element edge, the evaluation of the nearly singular integrals arising in adjacent elements requires a special treatment ${ }^{16 ; 17 ; 18}$. On the other hand, results deteriorate as interior points are chosen closer to element centers. Francis makes this choice of using the element centers as the only collocation points for the normal derivative integral formulation ${ }^{19}$. To avoid this problem, most 
implementations use piecewise-constant basis functions to allow using element centers as interior collocation points ${ }^{20 ; 21}$. Marburg and Amini consider 9-node conforming elements and perform a partial combination whereby the HIE and its normal derivative are combined only when the collocation point is the element center node, a formulation that is however only valid for this type of element ${ }^{22}$.

Few authors have considered formulations involving hypersingular operators that are applicable with usual $C^{0}$ conforming elements. Chien et al. propose a special treatment of the hypersingular kernel from known Laplace solutions ${ }^{23}$. Liu and Rizzo compare several kinds of elements (conforming, non-conforming and Overhauser $C^{1}$ continuous elements) and conclude that conforming elements give good results even though they do not satisfy the Lyapunov condition at the nodes ${ }^{24}$. An excellent survey of these methods is presented by Rego Silva ${ }^{25}$. In another paper ${ }^{26}$, Rego Silva and Wrobel present a new family of continuous/discontinuous three-dimensional boundary elements. Yan et al. introduce a new concept of discretized operator matrix to compute the operator matrix corresponding to the second derivative of the static Green's function ${ }^{27}$. This approach is only valid for smooth surfaces and numerical examples are performed on spheroid or prolate spheroid geometries.

The motivation for this paper is to propose a robust and efficient computational scheme for the CHI method valid for smooth as well as piecewise-smooth surfaces, based on an indirect procedure exploiting discretized versions of the Calderón identities linking the integral operators for associated Laplace problems. Our proposed approach is presented for $C^{0}$-conforming elements, since they can be used here even though the BEM-discretized surface does not satisfy the Lyapunov condition ${ }^{24}$ at the nodes; it is however equally applicable to non-conforming (i.e. discontinuous) boundary element approximations. Only weakly singular integrals and corresponding standard quadrature rules are needed for the numerical implementation. We compare the Burton and Miller method and the Schenck method on several configurations: sphere (smooth, convex), peanut (smooth, non-convex), cube (non-smooth, convex) and cat's eye (non-smooth, non-convex). Additionally, a regularized version $\alpha=i k /\left(k^{2}+\lambda\right)$ of the classical BM coupling factor $\alpha=i / k$ is proposed.

\section{The Burton and Miller formulation}

For an unbounded acoustic medium (with wave velocity $c$ ) occupying the exterior of a closed surface $\Gamma \subset \mathbb{R}^{3}$, we consider the acoustic Neumann exterior problem whereby the normal derivative of the acoustic pressure $p$ is given: $\partial_{n} p=g$ on $\Gamma$ with $g$ known. The HIE formulation for this problem reads

$$
\kappa^{+}(P) p(P)=\iint_{\Gamma}\left[\frac{\partial G_{k}(P, Q)}{\partial_{n_{Q}}} p(Q)-\frac{\partial p(Q)}{\partial_{n_{Q}}} G_{k}(P, Q)\right] \mathrm{d} Q
$$

where $P$ is the field point, $Q$ the source point on $\Gamma$, and $n_{Q}$ the outward-directed normal to $\Gamma$ at $Q$. The coefficient $\kappa^{+}(P)$ is given by $\kappa^{+}(P)=1-\kappa^{-}(P)$, with

$$
\kappa^{-}(P)=-\iint_{\Gamma} \frac{\partial G_{0}(P, Q)}{\partial_{n_{Q}}} \mathrm{~d} Q
$$

In particular, $\kappa^{+}(P)=1$ for $P$ outside of $\Gamma, \kappa^{+}(P)=0$ for $P$ inside $\Gamma$, and $\kappa^{+}(P)=\kappa^{-}(P)=1 / 2$ at any smooth point $P$ of $\Gamma$. $G_{k}$ is the radiating free-space Green's function for the Helmholtz equation, given by

$$
G_{k}(P, Q)=\frac{e^{\mathrm{i} k|P-Q|}}{4 \pi|P-Q|},
$$


where $k=\omega / c$. The time-harmonic factor $e^{-\mathrm{i} \omega t}$ is, as usual, implicit in (3) and all other field quantities. Finally, for later reference, $G_{0}$ is the free-space Green's function for the Laplace equation:

$$
G_{0}(P, Q)=\frac{1}{4 \pi|P-Q|} .
$$

Equation (1) is first used for $P \in \Gamma$ as an integral equation for the unknown surface pressure $p$, and then provides an integral representation for the pressure at any evaluation point $P$ outside $\Gamma$. However, it is well-known that (1) as an integral equation for $p$ is singular at certain discrete values $k_{D}$ of $k$, namely the characteristic (or resonance) wave numbers for which the interior Dirichlet problem is not uniquely solvable. As a result, (1) does not have a unique solution for these frequencies. To restore solution uniqueness, Burton and Miller ${ }^{4}$ introduced a second equation by differentiating $(1)$ in the normal direction at $P$ :

$$
\kappa^{+}(P) \frac{\partial p(P)}{\partial_{n_{P}}}=\frac{\partial}{\partial_{n_{P}}} \iint_{\Gamma}\left[\frac{\partial G_{k}(P, Q)}{\partial_{n_{Q}}} p(Q)-G_{k}(P, Q) \frac{\partial p(Q)}{\partial_{n_{Q}}}\right] \mathrm{d} Q .
$$

As an integral equation for the unknown surface pressure $p$, Eq. (5) is singular for those discrete values $k_{N}$ of $k$ for which the interior Neumann problem is not uniquely solvable. Importantly, one always has $k_{D} \neq k_{N}$. Burton and Miller show that the following combination of integral equations (1) and (5) yields a unique solution $p$ for all frequencies

$$
\begin{aligned}
\kappa^{+}(P)\left[p(P)+\alpha \frac{\partial p(P)}{\partial_{n_{P}}}\right]=\iint_{\Gamma}\left[\frac{\partial G_{k}(P, Q)}{\partial_{n_{Q}}} p(Q)-\frac{\partial p(Q)}{\partial_{n_{Q}}} G_{k}(P, Q)\right] \mathrm{d} Q \\
\quad+\alpha \frac{\partial}{\partial_{n_{P}}} \iint_{\Gamma}\left[\frac{\partial G_{k}(P, Q)}{\partial_{n_{Q}}} p(Q)-G_{k}(P, Q) \frac{\partial p(Q)}{\partial_{n_{Q}}}\right] \mathrm{d} Q
\end{aligned}
$$

where the coupling parameter $\alpha$ is such that $\operatorname{Im}(\alpha) \neq 0$ when $k$ is real.

However, equation (6) introduces an integral operator whose kernel function $\partial_{n_{P}} \partial_{n_{Q}} G_{k}(P, Q)$ has a $|P-Q|^{-3}$ singularity. In the next section, we present a method which can be used to reformulate (5), and hence (6), for any type of boundary element.

\section{Reformulation of the normal derivative equation}

\section{A. Partially regularized equation}

We start from the following partially regularized reformulation of (5):

$$
\begin{aligned}
\kappa^{+}(P) \frac{\partial p(P)}{\partial_{n_{P}}}=\iint_{\Gamma} \frac{\partial^{2}\left[G_{k}(P, Q)-G_{0}(P, Q)\right]}{\partial_{n_{P}} \partial_{n_{Q}}} p(Q) \mathrm{d} Q+\frac{\partial}{\partial_{n_{P}}} \iint_{\Gamma} \frac{\partial G_{0}(P, Q)}{\partial_{n_{Q}}} p(Q) \mathrm{d} Q \\
\\
-\iint_{\Gamma} \frac{\partial\left[G_{k}(P, Q)-G_{0}(P, Q)\right]}{\partial_{n_{P}}} \frac{\partial p(Q)}{\partial_{n_{Q}}} \mathrm{~d} Q-\iint_{\Gamma} \frac{\partial G_{0}(P, Q)}{\partial_{n_{P}}} \frac{\partial p(Q)}{\partial_{n_{Q}}} \mathrm{~d} Q .
\end{aligned}
$$

In this equation, the kernels $\partial_{n_{P}} \partial_{n_{Q}}\left[G_{k}(P, Q)-G_{0}(P, Q)\right]$ and $\partial_{n_{P}}\left[G_{k}(P, Q)-G_{0}(P, Q)\right]$ are weakly singular and nonsingular, respectively, for $P, Q \in \Gamma$. Equation (7) defines a partially regularized formulation, because the hypersingularity singularity is transferred to derivative of the static Green's function $G_{0}$. When multiple values of $\omega$ are considered, e.g. for computing transient responses by Fourier synthesis, the static singular terms need only be computed once as they are frequency-independent. To do so, we use matrix operations, like Yan et al. ${ }^{27}$, rather than an analytical formulation of the corresponding integrals. 


\section{B. Discretization}

Upon introducing a conforming isoparametric boundary element discretization ${ }^{28 ; 29}$ involving $N$ nodes, Eq. (7) takes the form:

$$
K^{+} \partial_{n_{P}} p=\left[N_{k}-N_{0}\right] p+N_{0} p-\left[M_{k}-M_{0}\right] \partial_{n_{P}} p-M_{0} \partial_{n_{P}} p,
$$

where the matrices $\left[N_{k}-N_{0}\right],\left[M_{k}-M_{0}\right], N_{0}, M_{0}$ and $K^{+}$are defined by

$$
\begin{aligned}
{\left[N_{k}-N_{0}\right]_{i j} } & =\sum_{e \in E_{j}} \iint_{\Gamma_{\text {ref }}} \frac{\partial^{2}\left[G_{k}\left(P_{i}, Q_{e}(\boldsymbol{\xi})\right)-G_{0}\left(P_{i}, Q_{e}(\boldsymbol{\xi})\right)\right]}{\partial_{n_{P}} \partial_{n_{\boldsymbol{\xi}}}} N_{j}(\boldsymbol{\xi}) J_{e}(\boldsymbol{\xi}) \mathrm{d} \boldsymbol{\xi} \\
{\left[M_{k}-M_{0}\right]_{i j} } & =\sum_{e \in E_{j}} \iint_{\Gamma_{\mathrm{ref}}} \frac{\partial\left[G_{k}\left(P_{i}, Q_{e}(\boldsymbol{\xi})\right)-G_{0}\left(P_{i}, Q_{e}(\boldsymbol{\xi})\right)\right]}{\partial_{n_{P}}} N_{j}(\boldsymbol{\xi}) J_{e}(\boldsymbol{\xi}) \mathrm{d} \boldsymbol{\xi} \\
{\left[N_{0}\right]_{i j} } & =\sum_{e \in E_{j}} \iint_{\Gamma_{\mathrm{ref}}} \frac{\partial^{2} G_{0}\left(P_{i}, Q_{e}(\boldsymbol{\xi})\right)}{\partial_{n_{P}} \partial_{n_{\boldsymbol{\xi}}}} N_{j}(\boldsymbol{\xi}) J_{e}(\boldsymbol{\xi}) \mathrm{d} \boldsymbol{\xi} \\
{\left[M_{0}\right]_{i j} } & =\sum_{e \in E_{j}} \iint_{\Gamma_{\mathrm{ref}}} \frac{\partial G_{0}\left(P_{i}, Q_{e}(\boldsymbol{\xi})\right)}{\partial_{n_{P}}} N_{j}(\boldsymbol{\xi}) J_{e}(\boldsymbol{\xi}) \mathrm{d} \boldsymbol{\xi} \\
K_{i j}^{+} & =\kappa^{+}\left(P_{i}\right) \delta_{i j} .
\end{aligned}
$$

In these equations, $\Gamma_{\text {ref }}$ is the reference element used in the isoparametric formulation, $\boldsymbol{\xi} \in \mathbb{R}^{2}$ is the parametric coordinate on $\Gamma_{\text {ref }}$, the mapping $\boldsymbol{\xi} \in \Gamma_{\text {ref }} \rightarrow Q_{e}(\boldsymbol{\xi}) \in \Gamma_{h}$ defines the geometry of the $e$-th element of the approximate surface $\Gamma_{h}, J_{e}$ is the Jacobian of that mapping, and $N_{j}$ is the shape function associated with node $P_{j}$, used for interpolating both the geometry and the acoustic quantities. Any $C^{0}$-conforming element may be used; in practice, linear or quadratic shape functions are most often chosen. Moreover, $E_{j}$ is the list of all elements that make up the support of the shape function $N_{j}$ on $\Gamma_{h}$. The evaluation of the normal $n_{P}$ at a node $P$ depending on which element $P$ is considered to belong to, a reasonable approach consists in defining $n_{P}$ by averaging normals relative to all adjacent elements at $P$.

\section{Evaluation of $N_{0}$ and $M_{0}$}

We now propose an indirect approach for evaluating matrices $N_{0}$ and $M_{0}$. For this purpose, we note that any $p, \partial_{n} p$ solving the interior Laplace problem verify the (discretized) integral equations

$$
\begin{aligned}
K^{-} p & =-D_{0} p+S_{0} \partial_{n} p \\
K^{-} \partial_{n} p & =-N_{0} p+M_{0} \partial_{n} p
\end{aligned}
$$

where $S_{0}$ and $D_{0}$ are the matrices associated with the single- and double-layer integral operators for the Laplace equation:

$$
\begin{aligned}
{\left[S_{0}\right]_{i j} } & =\sum_{e \in E_{j}} \iint_{\Gamma_{\mathrm{ref}}} G_{0}\left(P_{i}, Q_{e}(\boldsymbol{\xi})\right) N_{j}(\boldsymbol{\xi}) J(\boldsymbol{\xi}) \mathrm{d} \boldsymbol{\xi} \\
{\left[D_{0}\right]_{i j} } & =\sum_{e \in E_{j}} \iint_{\Gamma_{\mathrm{ref}}} \frac{\partial G_{0}\left(P_{i}, Q_{e}(\boldsymbol{\xi})\right)}{\partial_{n_{\boldsymbol{\xi}}}} N_{j}(\boldsymbol{\xi}) J(\boldsymbol{\xi}) \mathrm{d} \boldsymbol{\xi}
\end{aligned}
$$

Solving (10a) for $\partial_{n} p$ and inserting the result $\partial_{n} p=S_{0}^{-1}\left[D_{0}+K^{-}\right] p$ into (10b), we obtain

$$
N_{0} p=\left[M_{0}-K^{-}\right] S_{0}^{-1}\left[D_{0}+K^{-}\right] p
$$


This equation holds for any $p$, so the operator $N_{0}$ is given by

$$
N_{0}=\left[M_{0}-K^{-}\right] S_{0}^{-1}\left[D_{0}+K^{-}\right]
$$

By similar reasoning on the Laplace exterior problem, we find

$$
N_{0}=\left[M_{0}+K^{+}\right] S_{0}^{-1}\left[D_{0}-K^{+}\right]
$$

Equations (12) and (13) imply equality of their right-hand sides. Upon rearrangement and using that $K^{+}+K^{-}=I$, the resulting equation yields the following expression for $M_{0}$ :

$$
M_{0}=S_{0}^{-1} D_{0} S_{0}-K^{+} S_{0}^{-1} K^{+} S_{0}+K^{-} S_{0}^{-1} K^{-} S_{0}
$$

It is important to note that $M_{0}$ and $N_{0}$ are evaluated in terms of quantities which involve only weakly singular integrals, namely $K^{+}, K^{-}, D_{0}$ and $S_{0}$. These terms only have to be computed once, using a standard BEM code with $k=0$. The matrix $M_{0}$ is computed first, then $N_{0}$ is evaluated by using (14) in either (12) or (13). Performing either derivation and recalling again that $K^{+}+K^{-}=I$ yields

$$
N_{0}=S_{0}^{-1}\left(D_{0}+K^{-}\right)\left(D_{0}-K^{+}\right)=S_{0}^{-1}\left(D_{0}-K^{+}\right)\left(D_{0}+K^{-}\right)
$$

In particular, when $\Gamma$ is a smooth surface (in which case $K^{-}=K^{+}=I / 2$ ), we find

$$
N_{0}=S_{0}^{-1}\left[D_{0}^{2}-\frac{1}{4} I\right]
$$

which is the formulation given by Yan et $\mathrm{al}^{27}$. Then equations (14) and (12) (or (13)) can be seen as a generalization of their formulation.

Using the foregoing approach, the discretized form of the Burton-Miller integral equation (6) for the acoustic Neumann exterior problem is then

$$
\left[D_{k}-K^{+}+\alpha\left(\left[N_{k}-N_{0}\right]+N_{0}\right)\right] p=\left[S_{k}+\alpha\left(K^{+}+\left[M_{k}-M_{0}\right]+M_{0}\right)\right] g .
$$

Setting up the system (17) requires only nonsingular and weakly singular integrations. The frequency-independent terms $M_{0}$ and $N_{0}$ only have to be computed once, before being added to the (weakly singular) terms $\left[M_{k}-M_{0}\right]$ and $\left[N_{k}-N_{0}\right]$ for each frequency of interest.

Equations (14) and (15) are in fact discretized versions of the Calderón identities linking the integral operators associated with Laplace problems (see e.g. Nedelec ${ }^{1}$, Theorem 3.1.3). The Calderón identities are useful in many aspects of boundary integral formulations, such as preconditioning ${ }^{30}$.

\section{Partial combination for piecewise-smooth objects}

When the surface $\Gamma$ is only piecewise-smooth (due to edges and corners), we can only effect a partial combination of the integral equations (1) and (5) because the normal derivative of the pressure is not clearly defined at edge or corner collocation points. This partial combination has been tested successfully by several authors ${ }^{19 ; 22 ; 31}$, who report that forming such combinations at one-third of the original number of collocation points is sufficient to make the resulting system of equations well-conditioned.

The indirect formulation of Sec. III..C. can still be applied when $\Gamma$ is piecewise-smooth. Assume that the boundary element approximation of $\Gamma$ still involves $N$ nodes, which are also pressure nodes. Then, since the normal is not uniquely defined at edge or corner nodes, those 
nodes support correspondingly multiple values of $\partial_{n} p$, so that the mesh involves $M>N$ nodal values of $\partial_{n} p$. There are $Q<N$ nodes not located on corners or edges, at which nodal values of $\partial_{n} p$ are uniquely defined. The integral equation (1) for the exterior problem in discrete form is then

$$
K^{+} p=D_{k} p-S_{k} \partial_{n} p,
$$

where $S_{k}$ is a rectangular $N \times M$ matrix while $K^{+}$and $D_{k}$ are square $N \times N$ matrices.

Then, the discretized version of equation (5) restricted to the $Q$ collocation nodes $P$ where $\partial_{n} p$ is uniquely defined is

$$
K_{v}^{+} \partial_{n} p=\left(\left[N_{k}-N_{0}\right]+N_{0}\right) p-\left(\left[M_{k}-M_{0}\right]+M_{0}\right) \partial_{n} p
$$

where $K_{v}^{+}$is the rectangular $Q \times M$ matrix formed by expanding $K^{+}$as a $M \times M$ square matrix and retaining only the $Q$ lines corresponding to the nodes at which the normal is uniquely defined. $\left[N_{k}-N_{0}\right], N_{0}$ are $Q \times N$ matrices and $\left[M_{k}-M_{0}\right], M_{0}$ are $Q \times M$ matrices.

Adapting the procedure of Section III..C. to the relevant Laplace integral formulations yields

$$
M_{0}=S_{0}^{\dagger} D_{0} S_{0}-K_{v}^{+} S_{0}^{\dagger} K^{+} S_{0}+K_{v}^{-} S_{0}^{\dagger} K^{-} S_{0}
$$

(where $S_{0}^{\dagger}=S_{0}^{T}\left(S_{0} S_{0}^{T}\right)^{-1}$ is the pseudo-inverse of the rectangular $N \times M$ real-valued matrix $S_{0}$ ) and

$$
N_{0}=\left(M_{0}+K_{v}^{+}\right) S_{0}^{\dagger}\left(D_{0}-K^{+}\right) \text {, }
$$

or (equivalently)

$$
N_{0}=\left(M_{0}-K_{v}^{-}\right) S_{0}^{\dagger}\left(D_{0}+K^{-}\right) .
$$

Now, we can combine the $N$ equations (18) and the $Q$ equations (19) in Burton-Miller fashion. This corresponds to setting $\alpha=0$ for the $N-Q$ edge or corner collocation nodes.

The proposed indirect approach, while mainly presented for use with $C^{0}$-conforming boundary elements, is also applicable to discretizations using non-conforming elements. In that context, the partial combination technique of Sec. 4 is not needed if the same approximation spaces are used for $p$ and $\partial_{n} p$.

\section{Numerical implementation}

Tests aiming at fine-tuning the implementation of the formulation proposed in Sections III., IV. have been performed. For brevity, they are not presented in detail, and only the conclusions are reported. The tests were done for triangular elements, but quadrangular elements could also easily be implemented.

Implementing the numerical integrations was easy. For the nonsingular integrals, Lyness's quadrature rule for triangles is used ${ }^{32}$, while the Gauss-Radau quadrature rule (which concentrates quadrature points near the singularity in an appropriate way) is used for the weakly singular integrals ${ }^{33}$. The convergence of the integral evaluations with respect to the number of quadrature points is fast. In our tests, 19 points (Lyness's quadrature rule) are sufficient for regular integration, while 25 points $(5 \times 5)$ for each subdivision of a singular element were found adequate for weakly singular integrations. These amounts can be reduced when the collocation point is far from the integration element ${ }^{34}$, but we do not carry out this optimization here. A few element subdivision patterns have also been tested, but this proved not to be critical, so we finally subdivide each singular element into two triangles even if the singular point is at a vertex.

Finally, the normal $n_{P}$ in Eqs. 9 is the average of the element normals at $P$ for all elements sharing $P$. 


\section{Numerical simulations}

In this section, the method is used to solve wave problems with Neumann boundary conditions on the surface of the structure, which is quite common in practice. The gradient pressure or velocity can be measured directly with accelerometers or vibrometric lasers or calculated with an FEM code. For our simulations, we place a monopole of unit strength at a source point $X$ in the interior region surrounded by $\Gamma$, which generates the acoustic field $G_{k}(X, \cdot)$ in the entire space. The corresponding normal derivative $\partial_{n} G_{k}(X, \cdot)$ then defines the Neumann data for the exterior problems solved thereafter (i.e. $\left.g=\partial_{n} G_{k}(X, \cdot)\right)$. The computed pressure field $p$ on $\Gamma_{h}$ can then be compared to its analytical counterpart $G_{k}(X, \cdot)$ on $\Gamma$ by means of the following error function:

$$
E:=\left(\frac{\sum_{i=1}^{N}\left|p_{i}-G_{k}\left(X, Q_{i}\right)\right|^{2}}{\sum_{i=1}^{N}\left|G_{k}\left(X, Q_{i}\right)\right|^{2}}\right)^{1 / 2}
$$

where $Q_{i}$ are the $N$ nodes on $\Gamma$ and $p_{i}$ are the computed nodal pressures.

\section{A. Case configurations}

We consider four different geometrical configurations, thereafter referred to as Case 1 to 4 . All meshes are made of quadratic six-node (T6) triangles and created with GMSH ${ }^{35}$ (http://geuz.org/gmsh/).

In Case $1, \Gamma$ is a unit sphere, which is both smooth and convex. The mesh is composed of 2622 nodes and 1310 elements. The monopole is located at $X=\left(\begin{array}{lll}0 & 0 & 0.2\end{array}\right)$.

In Case 2, $\Gamma$ is a peanut-shaped surface, defined by the parametric representation

$$
\begin{array}{ll}
x & =\sqrt{\cos 2 \theta+\sqrt{1.5-\sin ^{2} 2 \theta}} \sin \theta \cos \gamma, \\
y & =\sqrt{\cos 2 \theta+\sqrt{1.5-\sin ^{2} 2 \theta}} \sin \theta \sin \gamma, \quad(0 \leq \theta \leq \pi, 0 \leq \gamma \leq 2 \pi) \\
z & =\sqrt{\cos 2 \theta+\sqrt{1.5-\sin ^{2} 2 \theta}} \cos \theta
\end{array}
$$

This surface is smooth but not convex (Fig.1b). The mesh is composed of 2294 nodes and 1146 elements. The monopole is located at $X=\left(\begin{array}{lll}0.2 & 0 & 0.01\end{array}\right)$.

In Case 3, $\Gamma$ is a unit cube, which is piecewise smooth and convex (Fig.1c). The mesh is composed of 2402 nodes and 1200 elements. The monopole is located at $X=(0.20 .10 .3)$.

Finally, in Case 4, $\Gamma$ is a cat's eye (unit sphere with one octant removed), which is piecewise smooth and non-convex (Fig.1d). This is a severe, often used, test case. The mesh is composed of 2338 nodes and 1168 elements. The monopole is located at $X=\left(\begin{array}{lll}-0.5 & 0 & 0\end{array}\right)$.

\section{B. Condition number}

Figure 2 plots the condition number of the governing matrix arising from BEM discretization of the HIE, CHIEF and BM formulations against the frequency for Cases 1 and 4 . For the HIE formulation, the condition number clearly shows peaks at frequencies such that $k=m \pi$ $(m=1,2 \ldots)$. Secondary peaks can also be seen; these appear due to the use of quadratic shape functions, a phenomenon previously observed by Marburg and Amini ${ }^{22}$. The CHIEF method, here used with 27 points for all frequencies (see Sec. C. for details on their arrangement), yields a condition number curve that is smooth only at low frequencies, evidencing the need for increasing the number of interior points with the frequency.

The condition number curve for the BM formulation with the classical value $\alpha=\mathrm{i} / k$ of the coupling factor is smooth at all frequencies. However, condition numbers are higher than those from the HIE formulation at low frequencies. Noting that the HIE gives good results at 


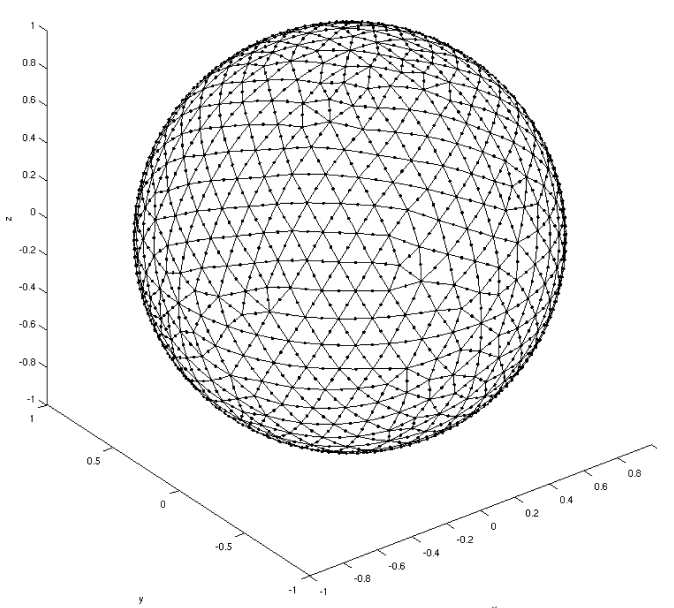

(a) Case 1: unit sphere (smooth, convex)

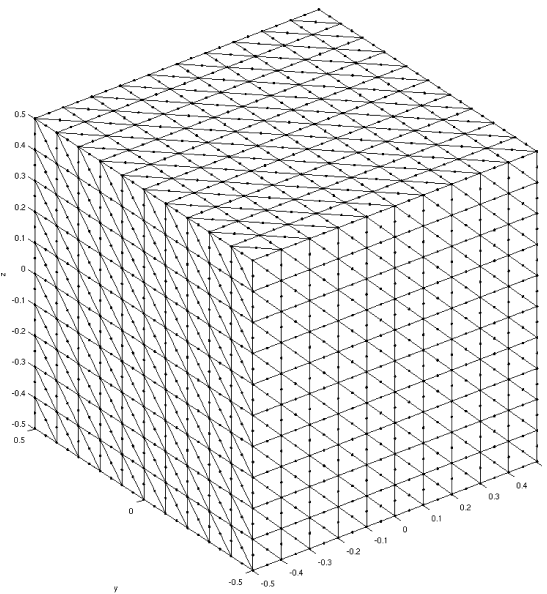

(c) Case 3: unit cube (non-smooth, convex)

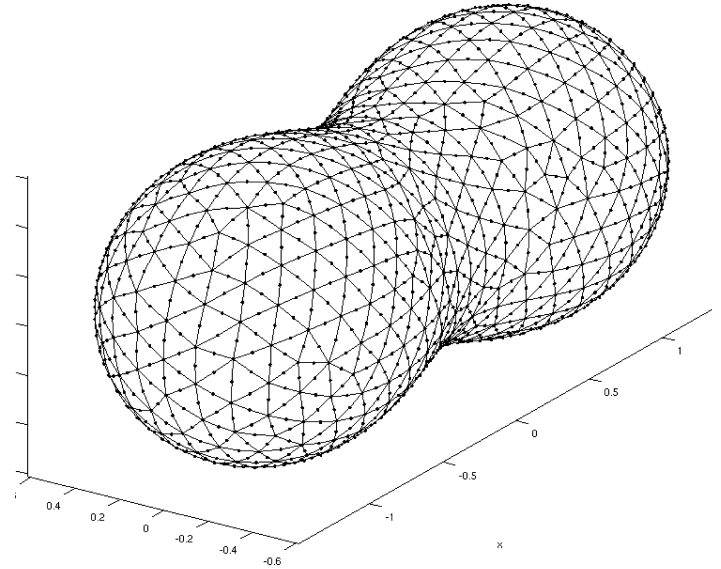

(b) Case 2: peanut (smooth, non-convex)

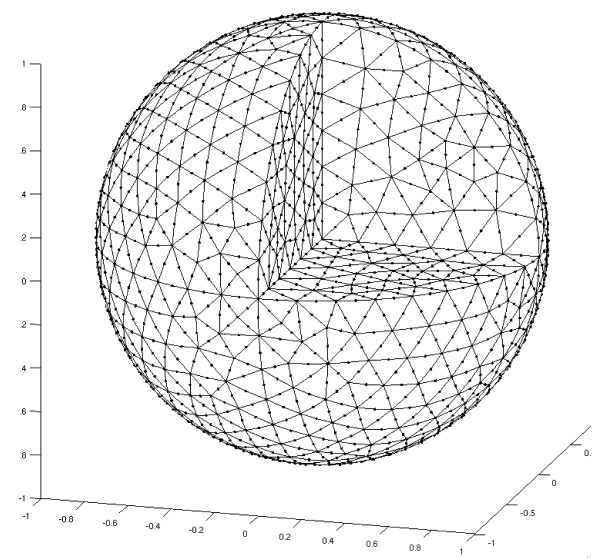

(d) Case 4: cat's eye (non-smooth, non-convex)

Figure 1: Test configurations
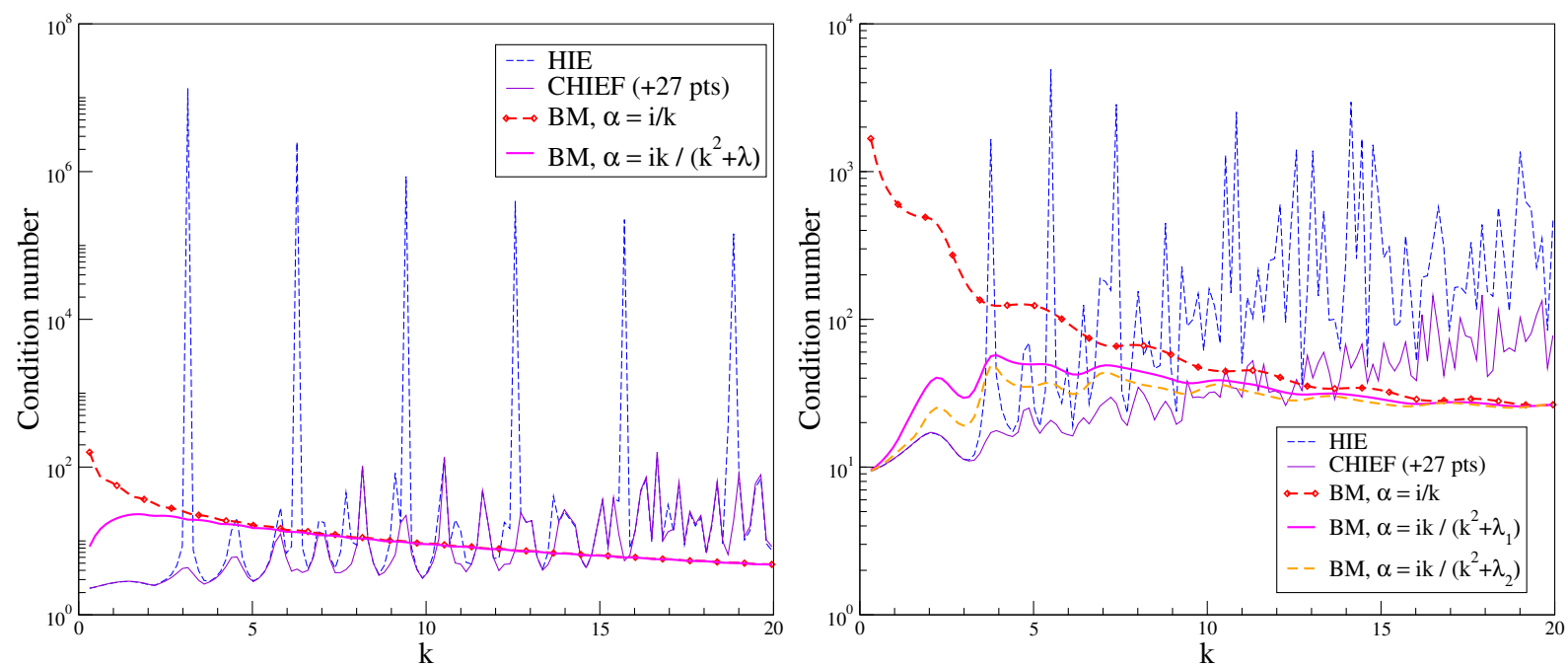

Figure 2: Condition number of the different formulations for Case 1 (sphere, left) and Case 4 (cat's eye, right). 
frequencies lower than the first fictitious eigenfrequency, for which $\alpha=\mathrm{i} / k$ is high, we consider the following modified coupling factor for the BM formulation, whose low-frequency behavior is regularized by means of the parameter $\lambda>0$ (with $\lambda=0$ restoring $\alpha=\mathrm{i} / k$ ):

$$
\alpha=\frac{\mathrm{i} k}{k^{2}+\lambda} .
$$

For Cases 1,2 involving smooth surfaces, we set $\lambda=\pi$ and $\lambda=4.55$ respectively and corresponding to the first fictitious eigenfrequencies of the HIE formulation. For non-smooth surfaces, $\lambda$ can be set to larger values. For example, we use $\lambda=15$ for Case 3 (cube) and $\lambda=30$ for Case 4 (cat's eye). The value chosen for $\lambda$ is not critical, provided that the condition number of the resulting matrix does not exceed that arising from the choice $\alpha=\mathrm{i} / k$; the results shown in Figure 2 (right) fulfil this requirement.

\section{Comparison of the Schenck and Burton-Miller methods}

For Cases 1,2 and 3, the CHIEF points are the 27 points of a $3 \times 3 \times 3$ regular cubic grid centered at the origin, the grid spacing being $0.25 \mathrm{~m}$ (which guarantees that all CHIEF points are interior). For Case 4 (cat's eye), the above set of points is translated by $(-0.350 .350) \mathrm{m}$, ensuring that all points are interior.

Figure 3 present the results for all four configurations. The present Burton-Miller formulation gives the best results at all frequencies for Case 1, and results similar to those of CHIEF at all frequencies for Case 2. For Cases 3 and 4, corresponding to surfaces with edges and corners, the CHIEF results are better than those produced by the present BM method at low frequencies, but become unsatisfactory at high frequencies for Case 3, possibly due to the (arbitrarily chosen) CHIEF points becoming inadequate in that case. The results given by the BM formulation are acceptably accurate in all cases, and their accuracy has, unlike those produced by CHIEF, a smooth, non-fluctuating dependence on the frequency.

\section{Comparison of linear and quadratic shape functions}

To see how the solution accuracy is improved by using quadratic shape functions (T6), we compare the relative errors obtained with corresponding results based on T3 shape functions with the same number of nodes (i.e. replacing each 6-noded quadratic triangular element by four 3-noded linear triangular element). Note that, for the same number of nodal unknowns, using T3 elements entails four times as many evaluations of element integrals. Figure 4 shows the improvement in solution accuracy (for both the CHIEF and Burton-Miller formulations) achieved by using T6 elements. This observation is consistent with the inferior performance of linear conforming elements reported in ${ }^{14}$.

\section{Conclusion}

Yan et al. introduced the concept of discretized operator matrix to compute the hypersingular integral involved in the composite Helmholtz integral equation proposed by Burton and Miller. They used double surface integrals, leading to a formulation that is valid only for smooth geometries. In our paper, the Laplace equations for the interior and exterior problems are used to compute the hypersingular operator $N_{0}$, but also the singular operator $M_{0}$. The method is valid for any kind of geometry and for higher-order element implementations and can be seen as a generalization of the Yan et al. formulation. The first advantage is the numerical implementation, which is very simple because it only requires a polar coordinate change and classical 


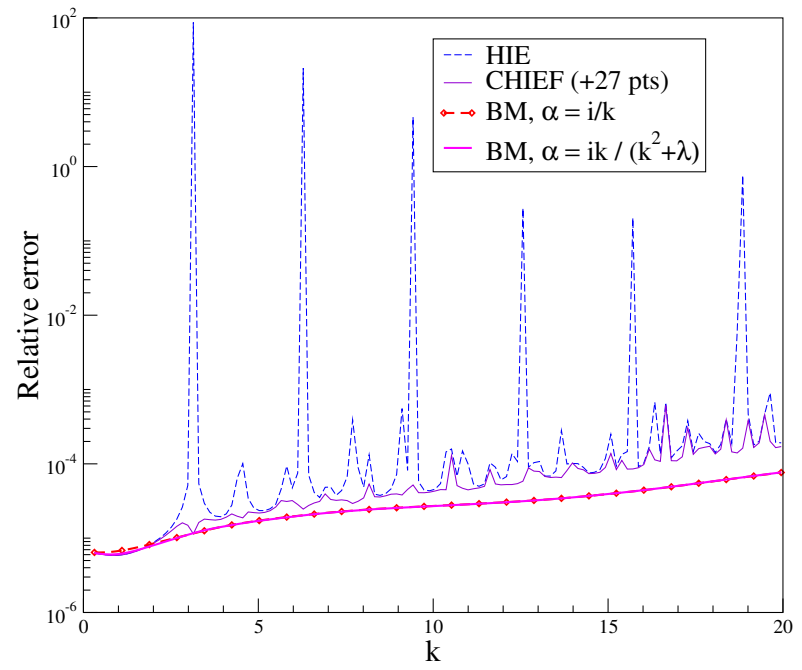

(a) Case 1 (sphere, $\lambda=\pi$ )

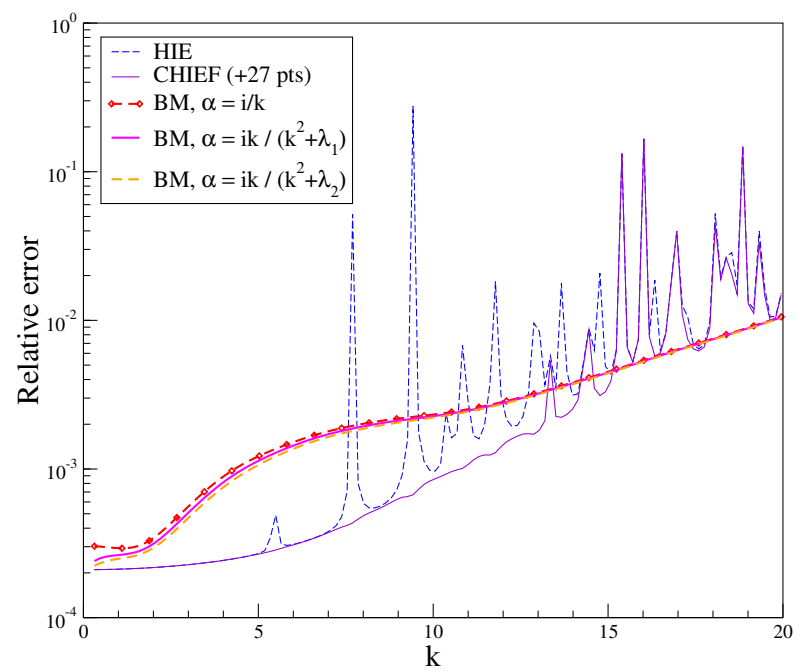

(c) Case 3 (cube, $\lambda_{1}=15, \lambda_{2}=30$ )

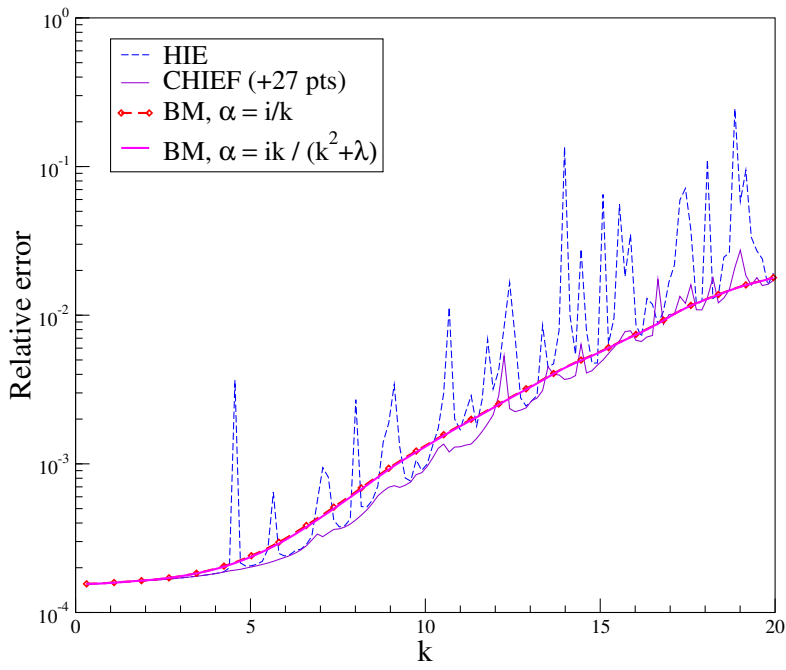

(b) Case 2 (peanut, $\lambda=4.55$ )

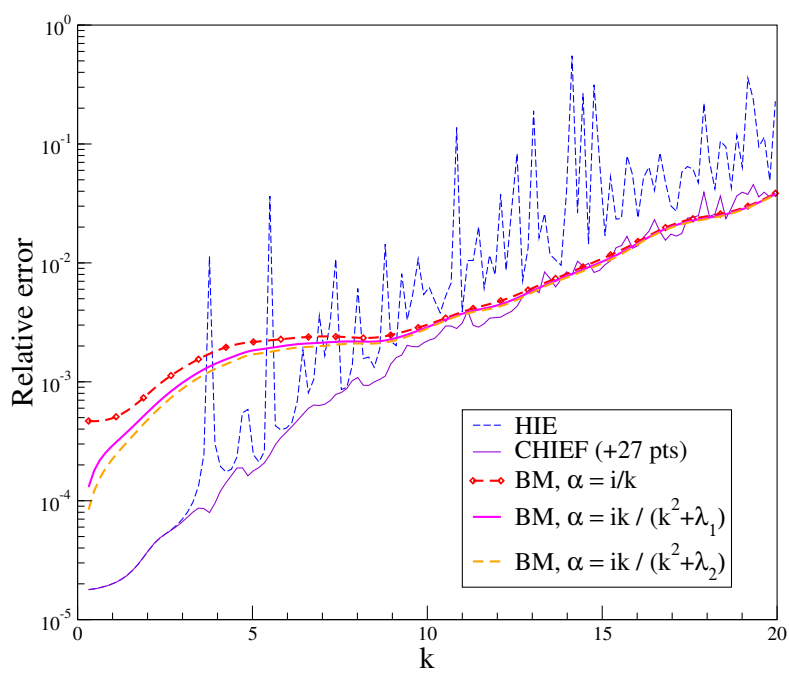

(d) Case 4 (cat's eye, $\lambda_{1}=15, \lambda_{2}=30$ )

Figure 3: Relative solution errors of the different formulations.

quadrature rules. The second advantage of the method is that the collocation points coincide with the nodal points of the mesh. The HIE and its normal derivative are combined directly at collocation nodes, unlike other methods which need interior element collocation points. When the surface is not smooth, a partial combination is used and is sufficient to give accurate results.

The CHIEF method and the Burton and Miller method are compared for four different configurations, including two involving non-smooth surfaces. CHIEF is found to give better results at low frequencies, where good interior points can easily be found. At higher frequencies, the Burton and Miller method is more reliable because finding good sets of interior points for the CHIEF method becomes difficult. The condition number produced by our BM approach is moderate at all considered frequencies, and consistently lower than that of CHIEF in the upper range of the considered frequencies.

Replacing the usual BM coupling factor $\alpha=\mathrm{i} / k$ with a version $\alpha=\mathrm{i} k /\left(k^{2}+\lambda\right)$ that is regularized at low frequencies improves the condition number at low frequencies without much influence on solution accuracy. The regularization parameter $\lambda$ can typically be set to the square of the value of the wave number corresponding to the first fictitious eigenfrequency of the 

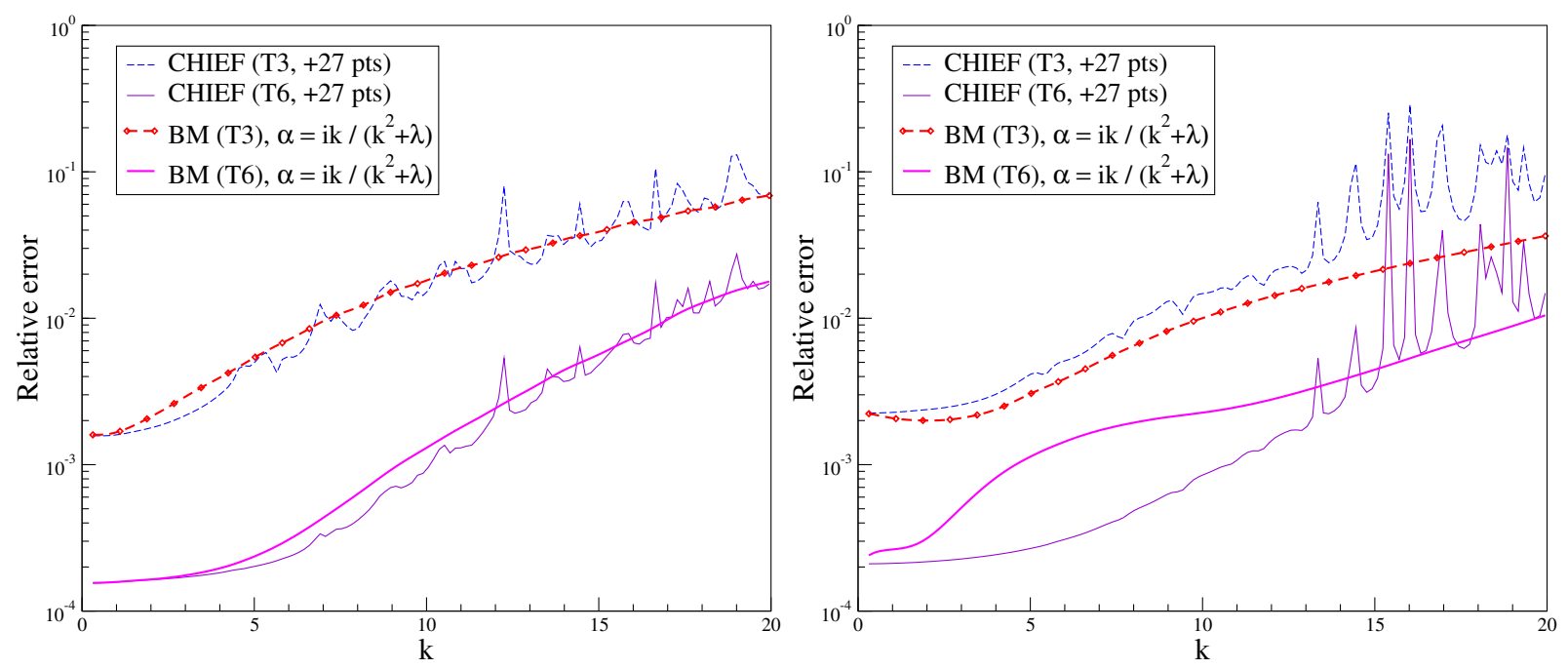

Figure 4: Comparison between T3 and T6 triangularizations for the sphere (left) and peanut (right) configurations $(\lambda=15)$.

HIE. The main additional computational cost of our BM approach lies in having to invert the Laplace single-layer operator (using the singular value decomposition when its pseudo-inverse is needed). However, this is only needed once for a given geometrical configuration, as this part of the computation is independent on the frequency.

\section{REFERENCES}

1. J. C. Nedelec, "Acoustic and electromagnetic equations: integral representations for harmonic problems", Springer (2001), 318 pages.

2. O. Steinbach, "Boundary integral equations for Helmholtz boundary value and transmission problems", in Direct and Inverse Problems in Wave Propagation and Applications (I. Graham, U. Langer, J. Melenk, M. Sini, editors), Radon Series on Computational and Applied Mathematics, vol. 14, pp. 253-292, Walter de Gruyter GmbH, Berlin/Boston (2013).

3. A. Schenck, "Improved integral formulation for acoustic radiation problems", J. Acoust. Soc. Am. 44 (1), 41-58 (1968).

4. A. J. Burton and G. F. Miller, "The Application of the Integral Equation Method to the Numerical Solution of Some Exterior Boundary Value Problems", Proc. R. Soc. London Ser. A 323, 201-210 (1971).

5. T. W. Wu and A. F. Seybert, "A weighted residual formulation for the CHIEF method in acoustics", J. Acoust. Soc. Am. 90 (3), 1608-1614 (1991).

6. C.-J. Zheng, H.-B. Chen, H.-F. Gao and L. Du, "Is the Burton-Miller Formulation really free of fictitious eigenfrequencies?", Eng. Anal. Bound Elem., 59, 43-51 (2015).

7. S. Marburg, "The Burton and Miller Method: Unlocking Another Mystery of Its Coupling Parameter", J. Comp. Acoust., 23, 1550016-1 (2015).

8. T.C. Lin, "A proof for the Burton and Miller integral equation approach for the Helmholtz equation", J. Math. Anal. Appl., 103, 565-574 (1984).

9. W. L. Meyer, W. A. Bell and B. T. Zinn, "Boundary integral solutions of three dimensional acoustic radiation problems", J. Sound Vib. 59, 245-262 (1978).

10. T. Terai, "On calculation of sound fileds around three dimensional objects by integral equation methods", J. Sound Vib. 69, 71-100 (1980).

11. I. C. Mathews, "Numerical techniques for three dimensional steady-state fluid-structure interaction", J. Acoust. Soc. Am. 79, 1317-1325 (1986).

12. T. W. Wu, A. F. Seybert and G. C. Wan, "On the numerical implementation of Cauchy principal 
value integral to insure a unique solution for acoustic radiation and scattering", J. Acoust. Soc. Am. 90 (1), 554-560 (1991).

13. K. A. Cunefare, G. Koopmann and K. Brod, "A boundary element method for acoustic radiation valid for all wavenumbers", J. Acoust. Soc. Am. 85 (1), 39-48 (1988).

14. S. Marburg and S. Schneider, "Influence of element types on numeric error for acoustic boundary elements", J. Comp. Acoust., 113, 363-386 (2003).

15. K. Chen, J. Cheng and P. J. Harris, "A new study of the Burton and Miller method for the solution of a 3D Helmholtz problem", IMA J. Appl. Math., 74, 163-177 (2009).

16. Y. Liu and F. J. Rizzo, "Scattering of elastic waves from thin shapes in three dimensions using the composite boundary integral equation formulation", J. Acoust. Soc. Am. 102(2), 926-932 (1997).

17. H. Ma and N. Kamiya, "Distance tranformation for the numerical evaluation of near singular boundary integrals with various kernels in boundary element method", Eng. Anal. Bound. Elem., 26, 329-339 (2002).

18. X. Qin, J. Zhang, G. Xie, F. Zhou and G. Li, "A general algorithm for the numerical evaluation of nearly singular integrals on 3D boundary element", J. Comp. Appl. Math., 235, 4174-4186 (2011).

19. D. T. I. Francis, "A gradient formulation of the Helmholtz integral equation for acoustic radiation and scattering", J. Acoust. Soc. Am. 93(4), 1700-1709 (1993).

20. A. V. Osetrov and M. Ochmann, "A fast and stable numerical solution for acoustic boundary element method equations combined with the Burton and Miller method for models consisting of constant elements", J. Comp. Acoust., 13(1), 1-20 (2005).

21. D.J. Chappell and P.J. Harris, "A Burton-Miller inverse boundary element method for near-field acoustic holography", J. Acoust. Soc. Am. 126(1), 149-157 (2009).

22. S. Marburg and Sia Amini, "Cat's eye radiation with boundary elements: comparative study on treatment of irregular frequencies", J. Comp. Acoust. 13(1), 21-45 (2005).

23. C.C. Chien, H. Rajiyah and S. N. Atluri, "An effective method for solving the hypersingular integral equations in 3-D acoustics", J. Acoust. Soc. Am. 88(2), 918-937 (1990).

24. Y. Liu and F. J. Rizzo, "A weakly singular form of the hypersingular boundary integral equation applied to 3-D acoustic wave problems", Comp. Meth. Appl. Mech. Eng. 96, 271-287 (1992).

25. J. J. Rego Silva, "Acoustic and elastic wave scattering using boundary elements", Topics in Engineering (vol. 18), Computational Mechanics Publications, Southampton Boston (1993), 134 pages.

26. J.J. Rego Silva and L.C. Wrobel, "A new family of continious/discontinious family of threedimensional boundary elements whith application to acoustic wave propagation", Int. J. Num. Meth. Eng., vol. 36, 1661-1679 (1993).

27. Z. Y. Yan, K. C. Hung and H. Zheng, "Solving the hypersingular boundary integral equation in three-dimensional acoustics using a regularization relationship", J. Acoust. Soc. Am. 113 (5), 26742683 (2003).

28. A. Seybert, B. Soenarko, F. J. Rizzo and D. J. Shippy, "An advance computational method for radiation and scattering of acoustic waves in three dimensions", J. Acoust. Soc. Am. 77 (2), 362-368 (1985).

29. M. Bonnet, "Boundary integral equation methods for solids and fluids", John Wiley and Sons, Chichester, 412 pages (1999).

30. O. Steinbach, W. L. Wendland, "The construction of some efficient preconditioners in the boundary element method", Advances Comput. Math. 9, 191-216 (1998).

31. P. J. Harris and S. Amini, "On the Burton and Miller boundary integral formulation of the exterior acoustic problem", ASME J. Vib. Acoust. 114, 540-546 (1992).

32. J. N. Lyness and D. Jespersen, "Moderate degree symmetric quadrature rules for the triangle", J. Inst. Math. Appl., 15, 19-32 (1975).

33. J. C. Lachat and J. O. Watson, "Effective numerical treatment of boundary integral equations: a formulation for three-dimensions elastostatics", Int. J. Num. Meth. Eng. 11, 1753-1768 (1976).

34. M. Rezayat, D. J. Shippy and F. J. Rizzo, "On time-harmonic elastic wave analysis by the boundary element method for moderate to high frequencies", Comp. Meth. Appl. Mech. Eng. 55, 349-367 (1986).

35. C. Geuzaine and J.-F. Remacle, "Gmsh: a three-dimensional finite element mesh generator with built-in pre- and post-processing facilities", Int. J. Num. Meth. Eng. 79, 1309-1331 (2009). 